\title{
Suppression by Snail
}

Epithelial-mesenchymal transition (EMT), mediated in part by the transcription factor snail (SNAI1), can increase cell invasion and metastasis. New insights into the relationship between cancer cells and host immunity during EMT have been provided by a study that shows that SNAI1 accelerates metastasis not only by enhancing invasion but also by inducing multiple immunosuppression mechanisms.

Chie Kudo-Saito and colleagues transduced B16-F10 murine melanoma cells with SNAI1, which led to an EMT-like phenotype. They found that the expression of the transcription factor forkhead box protein

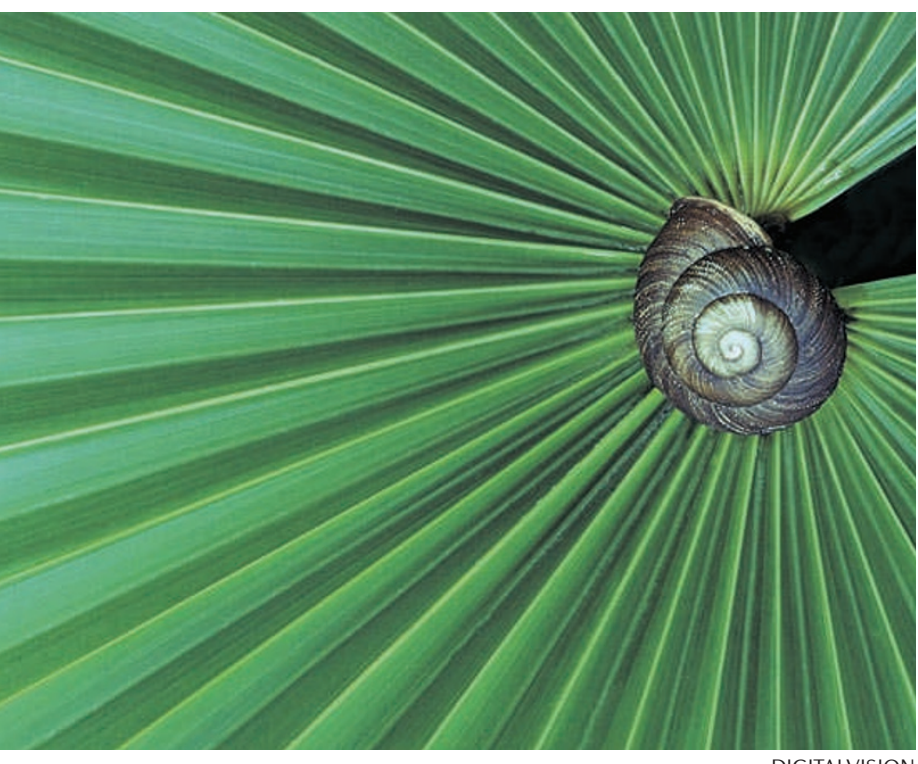

DIGITALVISION
P3 (FOXP3, an important mediator of the immunosuppressive function of regulatory $\mathrm{T}$ cells) was induced in the $\mathrm{CD}^{+}{ }^{+} \mathrm{T}$-regulatory $\left(\mathrm{T}_{\mathrm{Reg}}\right)$ cells present in a population of spleen cells (SPCs) co-cultured with SNAI1 ${ }^{+}$ tumour cells. The $\mathrm{CD} 4{ }^{+} \mathrm{FOXP}^{+}{ }^{+} \mathrm{T}_{\mathrm{Reg}}$ cells suppressed

$\mathrm{T}$ cell proliferation in the co-culture.

How are $\mathrm{T}_{\mathrm{Reg}}$ cells activated by cells that have undergone EMT? The authors examined the cytokines transforming growth factor- $\beta$ (TGF $\beta$ ), interleukin 10 (IL-10) and thrombospondin 1 (TSP1), which have been previously implicated in $\mathrm{T}_{\text {Reg }}$ cell induction, and found that the levels of TGF $\beta$ and TSP1 were significantly increased in SNAI1 ${ }^{+}$ melanoma cells. Treatment of SPC and $\mathrm{SNAI}{ }^{+}$tumour cell co-cultures with an anti-TSP1 monoclonal antibody reduced the induction of $\mathrm{CD}^{+}{ }^{+} \mathrm{FXX}^{+} \mathrm{T}_{\mathrm{Reg}}$ cells. Furthermore, natural induction of EMT by treatment with TGF $\beta$ or tumour necrosis factor- $\alpha$ (TNF $\alpha)$ also increased SNAI1 levels in B16-F10 cells and led to immunosuppression. The authors observed a similar relationship between naturally or SNAI1-induced EMT and immunosuppression in several human melanoma cell lines. They also observed the phenomenon in retinal pigment epithelial cells, suggesting that SNAI1-induced EMT might cause immunosuppression even in non-cancerous cells.
To study the role of EMT in immunosuppression in vivo, the authors transplanted SNAI1 ${ }^{+}$B16F10 cells into mice. The tumours formed by these cells showed increased metastasis compared with tumours from mock-transfected cells. The SNAI1 ${ }^{+}$tumours were resistant to immunotherapy, as were tumours originating from B16-F10 cells that had undergone TGF $\beta$ induced EMT. The authors injected small interfering RNA (siRNA) against Snail into SNAI1 ${ }^{+}$tumours and observed infiltration of immune cells and the induction of an anti-tumour immune response. Furthermore, Snail siRNA reduced tumour size and metastasis.

Intratumoural injection of an antiTSP1 antibody also induced a specific immune response and reduced tumour growth and metastasis, but to a lesser extent than Snail siRNA. Interestingly, the authors found that Snail siRNA or anti-TSP1 treatment also effectively inhibited the growth of mock-transfected tumours, suggesting that tumour cells with low levels of endogenous SNAI1 expression might also cause immunosuppression and that targeting SNAI1 in this case could still be beneficial. Meera Swami

ORIGINAL RESEARCH PAPER Kudo-Saito, C. et al. Cancer metastasis is accelerated through immunosuppression during Snail-induced EMT of cancer cells. Cancer Cell 15, 195-206 (2009) 